\title{
Propuesta de cuotas para conservación de un área natural protegida de México
}

\author{
Marco Antonio Almendarez-Hernández, Ismael Sánchez-Brito, \\ María Verónica Morales Zárate, César Augusto Salinas-Zavala*
}

\author{
Perfiles Latinoamericanos, 24(47) \\ 2016 | pp. 95-120 \\ DOI: $10.18504 / \mathrm{pl} 2447-007-2016$
}

\begin{abstract}
Resumen
La Reserva de la Biosfera Sierra La Laguna (RBSLA) es un área natural protegida. Como tal, constituye un instrumento de política ambiental que se deriva de las estrategias nacionales de conservación. Con el afán de mejorar los programas que la atienden, se propone incrementar la recaudación. Los métodos de preferencia pueden utilizarse como instrumento de manejo para controlar la futura demanda turística. Así, la capacidad de carga de esa Reserva no se verá rebasada y se podrá determinar al mismo tiempo una disposición a pagar (DAP) adicional. Ésta permitiría mayores fondos que coadyuvarían al cumplimiento de los objetivos de política ambiental. La DAP propuesta se estimó con los modelos probit del Método de Valoración Contingente (MVC) y los resultados muestran que la mediana de la DAP adicional varía entre 69 y 108 pesos por persona. Asimismo y con la entrada de los individuos a la Reserva, se pueden obtener beneficios adicionales de entre los 26 y 41 millones de pesos.
\end{abstract}

\begin{abstract}
The Sierra La Laguna Biosphere Reserve (RBSLA) is a protected area and as such, is an instrument of environmental policy derived from national conservation strategies, therefore, an increase in fund programs aimed at improving management actions to contribute to this objective. In this sense, stated preference methods can be used as a management tool to control future tourism demand and avoid exceeding the carrying capacity of the reserve, as well as to determine a willingness to pay (WтP) that could allow additional raise more funds to meet the objectives of environmental policy. WTP was estimated through Contingent Valuation Method using Probit model, our results showed that the median WTP varies between 69 and 108 pesos per person and can obtain additional economic benefits arising from the access of individuals to the Reserve which range between 26 and 41 million pesos.
\end{abstract}

Palabras clave: servicio ecosistémico, DAP, área natural protegida, biodiversidad, conservación, función de utilidad.

Keywords: ecosystem service, wTP, protected natural area, biodiversity, conservation, utility function.

* Marco Antonio Almendarez-Hernández es doctor en Ciencias Marinas y Costeras; adscrito al CiBnOR, es profesor-investigador en el Departamento Académico de Economía, Universidad Autónoma de Baja California Sur (UABCS). Ismael Sánchez Brito es doctor en Ciencias Marinas y Costeras; adscrito al CIBNOR, es profesor-investigador, uABCs. María Verónica Morales Zárate es doctora en Ciencias en el Uso, Manejo y Preservación de los Recursos Naturales y está adscrita a la Subordinación de Estudios Ambientales y Servicios Especializados (sEASE) del cibnor. César Augusto Salinas Zavala es doctor en Ecología Marina y coordinador de Vinculación, Servicios y Transferencia de Investigación (COVISTI), CIBNOR. 


\section{Introducción}

- U Unión Internacional para la Conservación de la Naturaleza (IUCN, por sus siglas en inglés) señala que las áreas protegidas (AP) son un instrumento estratégico de política ambiental para la conservación en todas las naciones. De acuerdo a la IUCN, un área protegida es un espacio geográfico claramente definido, reconocido, dedicado y gestionado, mediante medios legales u otros tipos de medios eficaces para conseguir la conservación a largo plazo de la naturaleza, de sus servicios ecosistémicos y sus valores culturales asociados. Al día de hoy, la IUCN define seis categorías de AP. De éstas, un $12.7 \% \mathrm{cu}-$ bre la superficie del planeta y el $1.6 \%$ corresponde al área oceánica (UNEPwCMC, 2012).

Ahora bien, en 2011 y en cuanto a los ingresos se refiere, los globales de exportación generados por el turismo receptor -incluido el transporte de pasajeros - superaron los 1200 billones de dólares. La cifra equivale a un promedio de 3.4 millones de dólares al día (omT, 2012). En 1998, se estimó que el turismo de naturaleza puede comprender entre 40 y $60 \%$ (Ecotourism Society, 1998), por ende, el estudio, discusión e investigación del fenómeno turístico y recreativo en las AP se han tornado recurrentes en foros de organismos internacionales. De hecho, se trata de una clase de turismo que cuenta ya con reconocimiento dentro de diversas convenciones y se le ha declarado como una oportunidad de desarrollo sustentable (CONANP, 2007).

En México y dentro de la Ley General de Equilibro Ecológico y Protección al Ambiente (LGEEPA), a las AP se las define como áreas naturales protegidas (ANP). La Secretaría de Turismo (sectur) ha encontrado que las anp son los destinos favoritos para desarrollar actividades de ecoturismo, turismo de naturaleza y de aventura (CONANP, 2007). Por su parte, la Comisión Nacional de Áreas Naturales Protegidas (CONAnP) estima que alrededor de 5.5 millones de turistas visitan cada año este tipo de lugares en todo el país y dejan una derrama económica de aproximadamente 274.72 millones de dólares por la prestación directa de servicios turísticos (CONANP, 2007).

El producto interno bruto turístico (PIBT) representa el valor de todas las actividades características y conexas del sector. Para 2012, éste participó con 8.4\% (116 203 millones de dólares) en la economía total (INEGI, 2014). La derrama económica turística en las ANP representa el $0.23 \%$ respecto al PIBT. Para el año 2012, se estimó que alrededor de 76 millones de turistas internacionales ingresaron al país (SECTUR, 2014). Si bien el turismo que visita las ANP no es en su totalidad foráneo, se calcula que un $6.5 \%$ de los turistas extranjeros realizan este tipo de visita. 
En su Programa Nacional de Áreas Naturales Protegidas (PNANP) 2007-2012, la CONANP declaró que una de sus estrategias es el turismo en esta clase de sitios, como parte del manejo de las Anp (CONANP, 2007). Por su parte, el segundo objetivo del Programa Sectorial de Turismo (PST) 2013-2018 versa sobre el fortalecimiento de las ventajas competitivas de la oferta turística. La finalidad sería impulsar la innovación, diversificación y consolidación de la oferta turística por región y destino. Asimismo, el quinto objetivo del PST se refiere al fomento del desarrollo sustentable de los destinos turísticos y de la ampliación de los beneficios sociales y económicos de las comunidades receptoras. Y una de las estrategias apunta a fortalecer la contribución del turismo a la conservación del patrimonio nacional y su uso sustentable (sECTUR, 2013).

Dentro del PNANP 2007-2012, una de las acciones es el desarrollo efectivo de instrumentos para el manejo de impactos sean económicos o de cobro de derechos. De hecho, se trata de herramientas tanto de recaudación como de control de la demanda turística.

El precio por el acceso a las ANP en México se establece en la Ley Federal de Derechos (DOF, 2012), y hay dos tarifas en tres categorías: a) marino de baja capacidad de carga, $b$ ) terrestre de baja capacidad de carga, y $c$ ) terrestre y marino que no se encuentren en las primeras dos. En los dos primeros, el precio por persona es de 4.43 dólares (53.97 pesos); en el tercer caso, es de 2.21 dólares (26.99 pesos). No obstante, el precio de cobro por el acceso a las ANP se estipula bajo condiciones de competencia imperfecta; en otras palabras, se desconoce la información completa del mercado — en este caso de los consumidores - y la disposición de pago por tener acceso a las áreas protegidas. A pesar de lo dicho, existe un instrumento útil para abordar este tipo de planteamientos: se trata del Método de Valoración Contingente (MVC). Éste mide tanto los valores de uso como los de no uso; un ejemplo es el de existencia y legado o herencia de recursos naturales propuesto por Krutilla (1967) y CVGM (1997). Cuando no existe mercado para los bienes o servicios públicos ambientales a valorar, o cuando se presentan fallas de mercado y es complicado asignar un precio, se recurre al Mvc. Éste busca determinar el valor monetario para bienes sin mercado en donde es necesario construirlo en forma hipotética (Carson 2000; Carson \& Hanemann, 2005).

En los últimos años, los países emergentes y en vías de desarrollo han aplicado el MvC para calcular el valor de los servicios culturales en áreas protegidas en los estudios de caso. El énfasis se ha puesto en tres categorías principales: parques marinos, parques terrestres y bosques, y especies emblemáticas (cuadro 1). Los estudios realizados han reportado una DAP entre 0.12 y 74.3 dólares, cifras que nos permitirán tener una mayor referencia en los resultados obtenidos en el presente estudio. De esta manera se evitará subestimar o sobreestimar la 
disposición a pagar (DAP) reportada en la Reserva de la Biosfera Sierra La Laguna (RBSLA).

Cuadro 1. Ejemplos de valoración de servicios ecosistémicos en áreas protegidas en economías emergentes y países en desarrollo

\begin{tabular}{|c|c|c|c|c|c|c|c|}
\hline Categoría & País & Área protegida & $\begin{array}{c}\text { Método } \\
\text { de } \\
\text { valoración }\end{array}$ & $\begin{array}{l}\text { Servicio } \\
\text { ecosistémico }\end{array}$ & $\begin{array}{c}\text { DAP } \\
\text { Estimada } \\
\text { en dólares }\end{array}$ & Concepto & Autor \\
\hline \multirow{5}{*}{$\begin{array}{l}\text { Parques } \\
\text { marinos }\end{array}$} & Vietnam & Nha Trang & MVC & $\begin{array}{l}\text { Servicios } \\
\text { recreativos }\end{array}$ & 0.61 & $\begin{array}{l}\text { Por visita } \\
\text { individual }\end{array}$ & $\begin{array}{l}\text { Lindsey } \\
\text { \& Holmes } \\
(2002)\end{array}$ \\
\hline & Filipinas & Anilao & MVC & $\begin{array}{l}\text { Servicios } \\
\text { recreativos }\end{array}$ & 3.70 & $\begin{array}{l}\text { Por visita } \\
\text { individual }\end{array}$ & $\begin{array}{l}\text { Arin y Kramer } \\
(2000)\end{array}$ \\
\hline & Filipinas & Mactan & MVC & $\begin{array}{l}\text { Servicios } \\
\text { recreativos }\end{array}$ & 5.50 & $\begin{array}{l}\text { Por visita } \\
\text { individual }\end{array}$ & $\begin{array}{l}\text { Arin \& Kramer } \\
(2001)\end{array}$ \\
\hline & Filipinas & Alona & MVC & $\begin{array}{l}\text { Servicios } \\
\text { recreativos }\end{array}$ & 3.40 & $\begin{array}{l}\text { Por visita } \\
\text { individual }\end{array}$ & $\begin{array}{l}\text { Arin \& } \\
\text { Kramer } \\
(2002)\end{array}$ \\
\hline & $\begin{array}{l}\text { Antillas } \\
\text { Holandesas }\end{array}$ & San Eustaquio & MVC & $\begin{array}{l}\text { Servicios } \\
\text { recreativos }\end{array}$ & 4 & $\begin{array}{l}\text { Por } \\
\text { inmersión } \\
\text { individual }\end{array}$ & $\begin{array}{l}\text { Riley et al. } \\
\text { (2006) }\end{array}$ \\
\hline \multirow{7}{*}{$\begin{array}{l}\text { Parques } \\
\text { terrestres y } \\
\text { bosques }\end{array}$} & Irán & $\begin{array}{l}\text { Parque Baba } \\
\text { Aman }\end{array}$ & MVC & $\begin{array}{l}\text { Servicios } \\
\text { recreativos }\end{array}$ & $5.31-9.43$ & $\begin{array}{l}\text { Por visita } \\
\text { individual }\end{array}$ & $\begin{array}{l}\text { Ghanbarpour } \\
\text { et al. (2011) }\end{array}$ \\
\hline & Malasia & Taman Negara & MVC & Ecoturismo & $2-6$ & $\begin{array}{l}\text { Por visita } \\
\text { individual }\end{array}$ & $\begin{array}{l}\text { Samdin } \\
(2008)\end{array}$ \\
\hline & Brasil & $\begin{array}{l}\text { Parque Estatal } \\
\text { Morro del Diablo }\end{array}$ & MVC & $\begin{array}{l}\text { Valor de } \\
\text { existencia }\end{array}$ & 60.39 & $\begin{array}{l}\text { Hectárea } \\
\text { por año }\end{array}$ & $\begin{array}{l}\text { Adams et al. } \\
(2008)\end{array}$ \\
\hline & Nepal & Annapurna & MVC & Ecoturismo & $69.2-74.3$ & $\begin{array}{l}\text { Por visita } \\
\text { individual }\end{array}$ & $\begin{array}{l}\text { Baral et al. } \\
(2008)\end{array}$ \\
\hline & Namibia & $\begin{array}{l}\text { Parques } \\
\text { nacionales }\end{array}$ & MVC & $\begin{array}{l}\text { Servicios } \\
\text { recreativos }\end{array}$ & $1-8.19$ & $\begin{array}{l}\text { Por visita } \\
\text { individual } \\
\text { al día }\end{array}$ & $\begin{array}{l}\text { Krug et al. } \\
(2002)\end{array}$ \\
\hline & Tailandia & $\begin{array}{l}\text { Sistema Nacio- } \\
\text { nal de Parques, } \\
\text { Doi Inthanon, Doi } \\
\text { Suthep y Mae Sa } \\
\text { Waterfall }\end{array}$ & MVC & $\begin{array}{l}\text { Servicios } \\
\text { recreativos }\end{array}$ & $0.12-1$ & $\begin{array}{l}\text { Por visita } \\
\text { individual }\end{array}$ & $\begin{array}{l}\text { Israngkura } \\
\text { (1998) }\end{array}$ \\
\hline & Costa Rica & $\begin{array}{l}\text { Parque } \\
\text { nacionales }\end{array}$ & MVC & $\begin{array}{l}\text { Servicios } \\
\text { recreativos }\end{array}$ & 8.85- 13.51 & $\begin{array}{l}\text { Por visita } \\
\text { individual }\end{array}$ & $\begin{array}{l}\text { Chase et al. } \\
\text { (1997) }\end{array}$ \\
\hline \multirow{4}{*}{$\begin{array}{l}\text { Especies } \\
\text { emblemáticas } \\
\text { (especies } \\
\text { amenazadas, } \\
\text { peligro de } \\
\text { extinción o } \\
\text { carismáticas) }\end{array}$} & Malasia & $\begin{array}{l}\text { Santuario de } \\
\text { Aves en Kapar }\end{array}$ & MVC & Conservación & $3.92-19.83$ & $\begin{array}{l}\text { Por visita } \\
\text { individual }\end{array}$ & $\begin{array}{l}\text { Amiry et al. } \\
(2009)\end{array}$ \\
\hline & Sri Lanka & $\begin{array}{l}\text { Protección del } \\
\text { elefante asiático }\end{array}$ & MVC & Conservación & $7-13.80$ & $\begin{array}{l}\text { Por visita } \\
\text { individual }\end{array}$ & $\begin{array}{l}\text { Bandara \& } \\
\text { Tisdell (2004) }\end{array}$ \\
\hline & China & $\begin{array}{l}\text { Conservación } \\
\text { del panda } \\
\text { gigante }\end{array}$ & MVC & Conservación & $0.49-15.15$ & $\begin{array}{l}\text { Por visita } \\
\text { individual }\end{array}$ & $\begin{array}{l}\text { Kontoleon } \\
\text { \& Swanson } \\
(2003)\end{array}$ \\
\hline & Camerún & $\begin{array}{l}\text { Conservación } \\
\text { del perro salvaje }\end{array}$ & MVC & Conservación & $0.10-1$ & $\begin{array}{l}\text { Por visita } \\
\text { individual }\end{array}$ & $\begin{array}{l}\text { Tsi et al. } \\
(2008)\end{array}$ \\
\hline
\end{tabular}

MVC=Método de Valor Contingente, MCV=Método Costo de Viaje

Fuente: Elaboración propia.

En México, la CONANP administra actualmente 176 ANP de carácter federal, lo cual representa más de 25 millones de hectáreas. De acuerdo con la LGEEPA

98 M. A. Almendarez-Hernández, I. Sánchez-Brito, M. V. Morales Zárate, C. A. Salinas-Zavala| Propuesta de cuotas para conservación de un área natural protegida de México | Perfiles Latinoamericanos, 24(47) | FLACso México | pp. 95-120 DOI: $10.18504 / \mathrm{pl} 2447-007-2016$ 
y su Reglamento, las ANP se clasifican en seis categorías; las más representativas son las reservas de la biosfera. El resto de las categorías son parques nacionales, monumentos naturales, áreas de protección de recursos naturales, áreas de protección de flora y fauna, y santuarios (CONAFOR, 2013).

Con el fin de valorar los servicios culturales en las ANP, se han realizado estudios empíricos. Al haber tenido acceso al Parque Nacional Cabo Pulmo, Baja California Sur (BCS), Muñoz et al. (2003) realizaron un estudio de la demanda turística por medio de encuestas y estadística descriptiva y hubo datos relevantes: la DAP promedio por entrar al parque fue de doce dólares por individuo. La mediana fue de diez y la máxima de veinticinco dólares. El costo del viaje promedio fue de 1789 dólares por persona. Para los turistas extranjeros los precios oscilaron entre 970 y 2800 dólares. Mientras que para los nacionales el promedio fue de 173 dólares.

En el Parque Nacional de Punta Cancún-Nizuc e Isla Mujeres, así como en el de Isla Contoy, Quintana Roo, Rivera \& Muñoz (2005) aplicaron el Mvc con el fin de estimar un precio óptimo para la entrada a los parques. En el primero estimaron un precio de entrada máximo de 7.98 dólares por persona; en el segundo 4.86 dólares por persona, para el caso de un monopolio simple. En el Parque Nacional de Isla Contoy calcularon un precio óptimo de 4.13 dólares por persona en temporada alta y 1.83 en temporada baja. A través del MVC, Casey (2006) estimó la DAP individual para la protección de corales en la Riviera Maya, Quintana Roo. El cálculo fue de entre veinte y ochenta dólares con un excedente del consumidor (EC) entre cien y cuatrocientos millones de dólares anuales. Al aplicar el MCv para el Parque Nacional de Loreto en Baja California Sur, Hernández et al. (2009) estimaron el EC en 10.44 millones de dólares. Con el Método Costo de Viaje (MCV), el máximo ajustado al 50\% fue de 23.32 millones de dólares.

Respecto a las especies emblemáticas, uno de los primeros trabajos fue el de Romo (1998), para la Reserva de la Biosfera de la Mariposa Monarca en el estado de Michoacán. Aquí estimó una DAP con MVC y con éste calculó un EC de entre 1.77 y 3.54 millones de dólares. La DAP estimada fue de 38.53 por familia. Asimismo, se aplicaron encuestas en el área metropolitana de la Ciudad de México; la DAP declarada promedio fue de 16.5 por familia. La aplicación del MVC en Estados Unidos declaró una DAP promedio de 16.08 por familia. A su vez, Pérez (1998) estimó los beneficios recreativos en la Reserva de la Biosfera de la Mariposa Monarca por medio del MCV. El EC promedio individual se estimó en 18.90 dólares y, por el total de visitantes promedio durante la temporada de hibernación, se obtuvo un EC agregado de 327 mil dólares.

En el presente trabajo se utilizó el Método de Valoración Contingente (MVC) para un posible ajuste en el cobro de derecho por el acceso a la Reserva de la 
Biosfera Sierra La Laguna (RBSLA) con base en la disposición a pagar (DAP) de la demanda potencial de los habitantes de los municipios de Los Cabos y La Paz. La finalidad es la obtención de recursos financieros que se traduzcan en acciones de mejora y conservación de la ANP conforme a su programa de manejo.

\section{Métodos}

\section{Área de estudio}

La Reserva de la Biosfera Sierra La Laguna (figura 1) se localiza en la parte sur de Baja California Sur, entre los municipios de La Paz y Los Cabos; cuenta con una extensión territorial de 112437 h. El 6 de junio de 1994 fue decretada como área protegida y es parte de un macizo montañoso de donde la Reserva ocupa una extensión de aproximadamente $48 \mathrm{~km}$ de largo y un promedio de $20 \mathrm{~km}$ de ancho (CONANP, 2003).

Figura 1. El polígono sombreado señala la Reserva de la Biosfera Sierra La Laguna

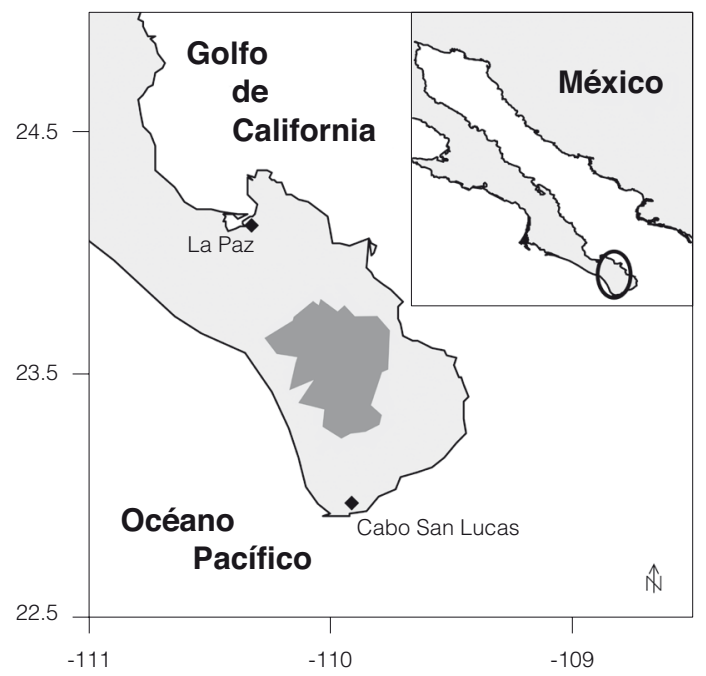

Fuente: Elaboración propia

La RBSLA se puede considerar como una "isla" de vegetación en el entorno árido, característico de la península de Baja California. Los cambios geológicos de la región de Los Cabos han sido factores determinantes para las condicio- 
nes climáticas, orográficas y biológicas de los ecosistemas que prevalecen en la zona. Como resultado directo de los procesos geológicos, la región presenta características de endemismo y se le considera como un centro de evolución reciente (Padilla et al., 1988). Por la composición del paisaje, uno de los principales atributos ambientales de la RBSLA es la vegetación, la cual es en sí misma un atractivo para visitar. Los factores físicos del ambiente favorecen el desarrollo de diferentes tipos de vegetación a lo largo de un gradiente altitudinal (Arriaga \& Ortega, 1988): a) Matorrales (sarcocaule y sarcocrasicaule), que se ubican desde el nivel del mar hasta los 500 metros de altitud. Los matorrales incluyen también al mezquital. Esta clase de vegetación alberga muchos elementos característicos del desierto sonorense. b) Selva baja caducifolia y subcaducifolia: distribuida de los 350 a los $800 \mathrm{~m}$ de altitud, cubre una extensión aproximada de 58701 hectáreas. c) Bosque de encinos: localizado entre los 800 y los $1200 \mathrm{~m}$ de altitud, todavía presenta muchos elementos tropicales en estratos inferiores al dosel superior. d) Bosque de pino-encino: se establece en las porciones más altas de la sierra. En la vertiente del Pacífico aparece a los $1400 \mathrm{~m}$, mientras que en la del golfo de California se ubica en los $1500 \mathrm{~m}$, y cubre alrededor de $8272 \mathrm{~h}$. e) Vegetación de galería: ubicada en los cañones a lo largo del gradiente altitudinal; f) Pastizal natural: ubicado principalmente en el área conocida como Valle de La Laguna (CONAnP, 2003).

La reserva cuenta con fauna muy diversa. Muchas de las especies — desde invertebrados hasta reptiles — son endémicas ${ }^{1}$ (cuadro 2 ).

\begin{tabular}{lc} 
Cuadro 2. Especies reportadas en la RBSLA \\
\hline Clasificación & Núm. especies \\
\hline Mastofauna & 47 \\
Ornitofauna & 59 \\
Insectos & 36 \\
Arácnidos & 61 \\
Herpetofauna & 48 \\
Total & 251 \\
\hline Fuente: Tomado de CONANP (2003).
\end{tabular}

1 Algunas especies endémicas de aves son el colémbolo (Xenyllodes mohuitli), aves como la pitorrea o carpintero bellotero (Melanerpes fomicivorus anguistifrons), el saltapalo (Sitta carolinesis lagunae), el vireo gorjeador (Vireo gilvus vistoriae) y el vireo solitario (Vireo solitarius lucasanus), la paloma pitayera (Zenaida asiática clara). Entre los reptiles se encuentran la salamanquesa (Phyllodactylus unctus), el ajolote (Xantusia vigilis gilberti), el bejori (Sceloporus licki y S. Hunsakeri). Y entre los mamíferos se hallan la musarańa (sorex ornatus lagunae), la tucita (Thomomys umbrinus alticolus) y el ratón pińonero (Peromyscus truei lagunae) 
El trabajo de campo se basó en encuestas personales. Las preguntas fueron de carácter dicotómico simple, con base en la recomendación de Hanemann (1994), quien argumenta que el entrevistado no conoce el límite de su disposición a pagar (DAP); por ello se prefirió el formato mencionado.

Para el diseño y aplicación del cuestionario se siguieron las recomendaciones del NOAA Panel (National Oceanic and Atmospheric Administration; Arrow et al., 1993). A fin de obtener una mayor precisión en la aplicación del método y minimizar los posibles sesgos, se recurrió a los sesgos de vehículo de pago (oECD, 1995), de diseño y de punto de partida (Boyle et al., 1985), al de operación (Cummings et al., 1994), al de información (Boyle \& Bishop, 1988; Bergstorm et al., 1989; Whitehead \& Blomquist, 1991; Hanley, 1988), al hipotético (Bishop \& Heberlein, 1979; Thayer, 1981), (Boyle et al., 1985) y al estratégico (Brookshire et al., 1976; Rowe et al., 1980; Hoehn \& Randall, 1987; Milon, 1989; Bergstorm et al., 1989; Mitchell \& Carson, 1989).

Para evitar los primeros cinco sesgos conocidos como instrumentales — que se originan por el uso de encuestas o del muestreo de la población - se necesita una adecuada estructuración de la encuesta (Osorio \& Correa, 2009). En la primera sección, el cuestionario debe contener un apartado introductorio, el cual, para conocimiento del entrevistado, deberá contener una descripción puntual del bien que se pretende valorar con base en los cambios que se lleven a cabo. En la segunda sección ha de exponerse una serie de preguntas al individuo para conocer las experiencias relacionadas sobre el bien ambiental, su percepción sobre el estado actual y futuro del mismo, así como las medidas que se podrían implementar para mejorar su cantidad y calidad. La tercera parte debe contener una valoración del bien. Aquí se plantea el escenario y la pregunta de vc por el bien ambiental. Se toca el punto del vehículo de pago, mismo que será el medio encargado de la recaudación. Finalmente, la última parte contiene la información sobre las características socioeconómicas del entrevistado que habrán de incluirse en el análisis econométrico (Riera, 1994).

Los pasos en el diseño de la encuesta, según Riera (1994), serían:

1) Claridad de lo que se busca medir en unidades monetarias.

2) Definición de la población relevante.

3) Definición del método más conveniente a utilizar.

4) Definición de modalidad de entrevista; a saber, si ésta será presencial, telefónica o por correo electrónico.

5) Selección de la muestra.

6) Redacción del cuestionario. 
7) Realización de entrevistas.

8) Captura de cuestionarios.

9) Explotación estadística de las respuestas.

10) Interpretación de los resultados obtenidos con base en el contexto de la investigación.

En este caso, la encuesta aplicada se compuso de tres elementos: a) uso de recursos, b) aspectos ambientales y $c$ ) datos socioeconómicos. La pregunta de la DAP se planteó bajo un escenario hipotético para mejorar la conservación de la RBSLA, a través de un cobro de cuota adicional por tener acceso a la zona. Se especificó que los fondos recaudados se destinarían a la ampliación y mejora del programa de manejo. El vehículo de pago propuesto fue mediante una cuota adicional que estableció la CONANP, misma que se traduciría en una tarifa que tendrían que pagar los que quisieran entrar a la reserva.

Los últimos dos sesgos pertenecen a la categoría de carácter hipotético y provienen del ejercicio propio de la Valoración Contingente (vc). Así es como surgen los "sesgos de no respuesta" que en los trabajos de vc se correlacionan con los factores que influyen en la disposición a pagar. Para solventar este problema, los investigadores recomiendan eliminar las respuestas cero. Con dicha supresión, desaparece este sesgo, conocido también como "respuestas de protesta” (Osorio \& Correa, 2009). En la aplicación del presente estudio no se presentó tal problema.

La muestra estimada para aplicar la encuesta se calculó a partir del número de habitantes mayores de 18 años de los municipios de La Paz y Los Cabos que, con base en el Conteo de Población y Vivienda de 2005 del InEgi, fue de 219 596 y 164162 habitantes, respectivamente. El total fue de 383758 habitantes. La selección de la muestra se basó en la preferencia de los turistas, ya que de aquí provienen para acceder a la RBSLA y estas visitas representan aproximadamente el 90\% del total. La muestra es representativa a un nivel de confianza del 95\% y un error de estimación del 4\%. En 2010 se levantaron 599 encuestas. El muestreo fue aleatorio simple.

\section{Método de Valoración Contingente y la respuesta de la DAP}

La investigación se sustenta en el Modelo Teórico de Utilidad Aleatoria (RUM, por sus siglas en inglés) para calcular la disposición a pagar utilizando como metodología el Mvc. El enfoque econométrico se basa en los modelos de opción discreta/continua (ODC) para analizar la relación de las variables que capturan las características de las personas encuestadas con la pregunta dictotómica de 
vc (Haab y McConell, 2002). Si seguimos este planteamiento, la función de utilidad se expresa de la siguiente manera:

$u_{i j}=u_{i}\left(y_{j}, z_{j}, \varepsilon_{i j}\right) \quad$ Donde $i=1,0$

Cuando $i$ toma el valor de 1, el programa de vc se implementa, o bien, la política en materia ambiental entra en acción y corresponde al estado final. Cuando se computa como 0, significa que se mantiene el statu quo. La utilidad del j-ésimo individuo está en función del ingreso, representado por $y_{j}$, donde $\mathrm{j}$ es el j-ésimo encuestado. Son también determinantes $z_{j}$, que representa un vector de variables socioeconómicas y otros atributos de la elección binaria; y $\varepsilon_{i j}$ considerado como un componente que incluye preferencias conocidas por el entrevistado, pero inobservables para el investigador.

Bajo esta perspectiva, si el individuo acepta pagar el precio por la operación del programa o la acción de la política, $i=1$ y la utilidad es $u_{1 j}$. Cuando $i=1$ significa que el entrevistado rechaza el precio por el escenario propuesto y su utilidad es $u_{0 j}$. En este sentido y en el proceso de valoración, el individuo se enfrenta a los dos escenarios que supone el aceptar pagar el precio y disfrutar del bien o no hacerlo y no tener acceso a éste. Sin embargo, hasta este momento sólo se conoce el cambio del statu quo $q^{0}$, al estado final $q^{1}$, en donde $q$ representa un bien o una mejora en la calidad ambiental para el bien considerado. Por lo tanto, la función de utilidad del estado final es mayor que la del statu quo y se representa de la forma siguiente:

$u_{1}\left(y_{j}-t_{j}, z_{j}, \varepsilon_{1 j}\right)>u_{0}\left(y_{j}, z_{j}, \varepsilon_{0 j}\right)$

Dicha superioridad de $u_{1}$ a $u_{0}$ se basa en la probabilidad que existe de que el entrevistado responda afirmativamente ante el pago en el escenario hipotético planteado, pues asume que, de hacerlo, estará en una mejor situación que de no realizarlo. Esto significa que la disposición a pagar del individuo por el cambio de $q^{0}$ a $q^{1}$ (es decir, en la mejora de la calidad ambiental), le genera un costo; en consecuencia, su nivel de ingreso disminuye. Según lo anterior, se genera un incremento en el nivel de utilidad del individuo a cambio de reducir su nivel de ingreso, ya que hay compensación al aumentar su bienestar a través de la mejora del bien ambiental. Así es como tiene lugar lo que se conoce como "variación compensada”.

Dado lo anterior, cuando el entrevistado j-ésimo responde afirmativamente por pagar el precio, es decir, cuando acepta el cambio en la mejora en la calidad ambiental, su probabilidad se expresa de la forma siguiente:

$\operatorname{Pr} o b\left(S i_{j}\right)=\operatorname{Pr} o b\left(u_{1}\left(y_{j}-t_{j}, z_{j}, \varepsilon_{1 j}\right)>u_{0},\left(y_{j}, z_{j}, \varepsilon_{0 j}\right)\right.$ 
Al descomponer la función de utilidad indirecta en un componente estocástico y otro determinista, ésta queda definida de la siguiente forma:

$u_{i}\left(y_{j}, z_{j}, \varepsilon_{i j}\right)=v_{i}\left(y_{j}, z_{j}\right)+\varepsilon_{i j}$

Transformándose la probabilidad de respuesta del entrevistado j en

$\operatorname{Pr} o b\left(S i_{j}\right)=\operatorname{Pr} o b\left[v_{1}\left(y_{j}-t_{j}, z_{j}\right)+\varepsilon_{1 j}>v_{0}\left(y_{j}, z_{j}\right)+\varepsilon_{0 j}\right]$

La ecuación 5 tiene dos componentes aleatorios y se pueden agregar en uno $\operatorname{como} \varepsilon_{i j}$. Si $F_{\varepsilon}(a)$ es la probabilidad de que la variable aleatoria $\varepsilon$ sea menor que (a), la probabilidad de obtener una respuesta positiva de parte del individuo $\mathrm{j}$ es la siguiente:

$\operatorname{Pr} o b\left(S i_{j}\right)=1-F_{\varepsilon}\left[v_{1}\left(y_{j}-t_{j}, z_{j}\right)-v_{0}\left(y_{j}, z_{j}\right)\right]$

Para analizar las respuestas de vc y obtener los coeficientes con la finalidad de estimar la DAP, se especificaron tres modelos: 1) lineal, 2) log-lineal y 3) Box-Cox. La limitante de la primera versión fue la suposición de que la utilidad marginal en el ingreso es constante para el escenario propuesto de vC y el statu quo, a menos que la alternativa de mejora ambiental proporcione un cambio sustancial. Bajo esta circunstancia, el precio ofrecido a los turistas es bajo y no genera un cambio significativo en su ingreso. Sin embargo, la modelación de casos consiste en cambios significativos en los ingresos de los entrevistados. La suposición de la linealidad no sería lo más recomendable cuando la lógica apunta hacia una utilidad marginal del ingreso decreciente. En este sentido, en la manipulación algebraica, el ingreso se anula y no da lugar a un rol en la elección discreta. Para tomar en cuenta diferencias en la disposición a pagar capturadas por el ingreso de los visitantes, se puede recurrir a una modelación con parámetros interactivos. Las variables se obtienen al multiplicar la cuota propuesta por variables binarias identificadas con ingresos bajos y altos.

El modelo log-lineal en el ingreso tiene como ventaja la relajación del supuesto de utilidad marginal constante en el ingreso de los individuos, lo que significa que la utilidad marginal del ingreso varía en las respuestas de vc ante cambios en el ingreso monetario. La limitante es que el valor esperado de la disposición a pagar no será el mismo que se obtenga con los modelos logit y probit como en la situación de la función de utilidad lineal. La ventaja de la tercera especificación es la obtención de mayor flexibilidad en su función con respecto al ingreso. La desventaja que presenta, sin embargo, es que la forma funcional 
de la función de utilidad puede considerarse como una "adivinanza educada" por parte del investigador, y es deseable tratar el parámetro fundamental $\lambda$ de la transformación Box-Cox como desconocido. La lista de variables y parámetros expresados en la pregunta de vc para las tres especificaciones de utilidad se presentan en el cuadro 3 .

Cuadro 3. Lista de variables y parámetros expresados en la pregunta de valoración contingente

\begin{tabular}{|c|c|}
\hline Variables & Definición \\
\hline$u$ & Utilidad del entrevistado \\
\hline$y$ & Ingreso del entrevistado \\
\hline$\varepsilon$ & Componente estocástico del modelo de regresión \\
\hline$q^{0}$ & Bien ambiental o calidad ambiental en el statu quo \\
\hline$q^{1}$ & Mejora en el bien ambiental o en la calidad ambiental \\
\hline$\sigma^{2}$ & Varianza del componente estocástico \\
\hline$\alpha$ & Parámetro asociado al vector de variables socioeconómicas \\
\hline$\beta$ & $\begin{array}{l}\text { Parámetro asociado a la variable contribución en dinero adicional a la tarifa que paga el } \\
\text { entrevistado por entrar a la RBSLA o precio sugerido }\end{array}$ \\
\hline$\lambda$ & Valor del parámetro de transformación Box-Cox $\varepsilon / \sigma$ \\
\hline$\theta$ & Es igual a la división entre el componente estocástico y la desviación estándar del error \\
\hline$\Phi(x)$ & Función de distribución normal estándar acumulada \\
\hline$z$ & Vector de variables socioeconómicas compuesto por: \\
\hline Monto-dap $(t)$ & $\begin{array}{l}\text { Contribución en dinero adicional a la tarifa que paga el entrevistado por entrar a la RBSLA o } \\
\text { precio sugerido al entrevistado }\end{array}$ \\
\hline $\begin{array}{l}\text { Dingresos } \\
\text { bajos*monto-dap }\end{array}$ & $\begin{array}{l}\text { Variable interactiva que consiste en multiplicar aquellas familias que tienen ingresos bajos, las } \\
\text { cuales toman el valor de } 1 \text { por sus respectivas contribuciones en dinero }\end{array}$ \\
\hline $\begin{array}{l}\text { Dingresos } \\
\text { altos*monto-dap }\end{array}$ & $\begin{array}{l}\text { Variable interactiva que consiste en multiplicar aquellas familias que tienen ingresos altos, las } \\
\text { cuales toman el valor de } 1 \text { por sus respectivas contribuciones en dinero }\end{array}$ \\
\hline pers_gas & Número de personas que aportan al gasto familiar \\
\hline encuen_r & $\begin{array}{l}\text { Toma el valor de } 1 \text { si el entrevistado sabía antes de llegar a la reserva donde se ubica la } \\
\text { RBSL y } 0 \text { de otro modo }\end{array}$ \\
\hline sabe_que & Toma el valor de 1 si el entrevistado sabe que es un ANP y 0 de otro modo. \\
\hline ser_am_c & $\begin{array}{l}\text { Variable categórica referente a la calificación que el entrevistado le otorga en importancia } \\
\text { a conservar la biodiversidad de la RBSL. Toma valores de } 1 \text { no es importante, } 2 \text { no es muy } \\
\text { importante, } 3 \text { es importante y } 4 \text { es muy importante. }\end{array}$ \\
\hline escolaridad & $\begin{array}{l}\text { Nivel de escolaridad del encuestado. Toma valores de } 0 \text { si no tiene ningún nivel de escolari- } \\
\text { dad, } 1 \text { si cuenta con la primaria, } 2 \text { secundaria, } 3 \text { preparatoria, } 4 \text { licenciatura y } 5 \text { si cuenta con } \\
\text { estudios de posgrado. }\end{array}$ \\
\hline imp_gen & $\begin{array}{l}\text { Variable categórica referente a la calificación que el entrevistado le otorga en dejarle un mejor } \\
\text { medio ambiente de la RBSL a futuras generaciones. Toma valores de } 1 \text { no es importante, } 2 \text { no } \\
\text { es muy importante, } 3 \text { es importante y } 4 \text { es muy importante. }\end{array}$ \\
\hline
\end{tabular}

Fuente: Elaboración propia con base en Habb \& McConell (2002).

106 M. A. Almendarez-Hernández, I. Sánchez-Brito, M. V. Morales Zárate, C. A. Salinas-Zavala | Propuesta de cuotas para conservación de un área natural protegida de México | Perfiles Latinoamericanos, 24(47) | FLAcso México | pp. 95-120 DOI: $10.18504 / \mathrm{pl} 2447-007-2016$ 
En la primera función, la utilidad del individuo que corresponde a la elección i puede definirse como una función lineal del ingreso y un vector que comprende variables socioeconómicas en la forma siguiente:

$v_{i j}\left(y_{j}\right)=\alpha_{i} z_{j}+\beta_{i}\left(y_{j}\right)$

Tal que $\alpha_{i}$ es un vector de parámetros.

Si se representa la utilidad del statu quo como $v_{0 j}\left(y_{j}\right)=\alpha_{0} z_{j}+\beta_{0}\left(y_{j}\right)$ y la utilidad del estado final como $v_{1 j}\left(y_{j}-t_{j}\right)=\alpha_{1} z_{j}+\beta_{1}\left(y_{j}-t_{j}\right)$, entonces, con su sustracción, se obtiene el cambio en la utilidad determinista:

$v_{1 j}-v_{0 j}=\left(\alpha_{1}+\alpha_{0}\right) z_{j}+\beta_{1}\left(y_{j}-t_{j}\right)-\beta_{0} y_{j}$

Si se agrupan los parámetros en $\alpha, \beta$, y si se incluye el componente aleatorio $\varepsilon_{j}$ la ecuación de probabilidad de que el individuo j conteste positivamente al cambio en la calidad ambiental es

$\left.\operatorname{Pr} o b\left(S i_{j}\right)=\operatorname{Pr} o b\left(\alpha z_{j}-\beta t_{j}+\varepsilon_{j}\right)>0\right)$

Una vez especificada la versión de la función de utilidad indirecta y terminada la captura de las encuestas, se procede al análisis de la pregunta de vc y del resto de la información con base en los modelos de ODC, como en los de elección discreta binaria. Para estimar los parámetros de la función de utilidad, se requiere especificar el tipo de distribución que sigue el componente estocástico $\varepsilon_{j}$. La distribución puede ser una normal tipificada o una distribución logística. Asimismo, se pueden utilizar funciones de masa de valor extremo. La estimación para obtener los coeficientes de regresión es por medio de la maximización de la función de verosimilitud:

$L=\prod_{Y_{i}=0} F(-X \beta) \prod_{Y_{i}=1}(1-F(-X \beta))$

Para nuestra investigación se utilizó el modelo probit, uno de los más recurrentes para estimar los parámetros de las variables relacionadas con la respuesta dicotómica de vc en el campo de la econometría. Las estimaciones del modelo logit se encuentran en el anexo. Éstas no se interpretan en el desarrollo del trabajo, pues de acuerdo con Train (2009), este tipo de tratamiento econométrico presenta serias limitaciones en su uso: 1 ) no puede representar la variación aleatoria de las preferencias al no admitir que las diferencias en éstas se relacionan con las características observadas de los entrevistados. Supone además que tienen la 
misma respuesta ante cambios en las variables independientes. 2) Presenta patrones de sustitución restrictivos debido a la propiedad de independencia de alternativas irrelevantes (IIA, por su acrónimo en inglés); y ya que implica sustitución proporcional entre alternativas, se requieren otros modelos para incluir formas más flexibles de sustitución. 3) No puede utilizarse con datos de panel cuando los factores no observados están correlacionados en el tiempo para cada sujeto que decide. La especificación probit toma en cuenta estas tres limitaciones: contempla la variación aleatoria de preferencias, permite cualquier patrón de sustitución y es aplicable a datos de panel con errores correlacionados temporalmente.

En el modelo probit, se supone que la función de distribución asociada a $\varepsilon$ sigue una distribución en forma normal e idéntica con media cero y varianza $\sigma^{2}$. Además, $\varepsilon_{j} \sim N\left(0, \sigma^{2}\right)$ y si $\theta=\varepsilon / \sigma$, entonces $\theta \sim N(0,1)$. Por lo tanto el modelo probit se expresa de la forma siguiente:

$\operatorname{Pr} o b\left(\varepsilon_{j}<\alpha z_{j}-\beta t_{j}\right)=\operatorname{Pr} o b\left(\theta<\frac{\alpha z_{j}}{\sigma}-\frac{\beta}{\sigma} t_{j}\right)=\Phi\left(\frac{\alpha z_{j}}{\sigma}-\frac{\beta}{\sigma} t_{j}\right)$

Donde $\Phi(x)$ significa la función normal estándar acumulada y la probabilidad se especifica como la división de los parámetros entre una varianza desconocida.

La función de utilidad log-lineal en el ingreso se puede estructurar de la forma siguiente:

$\left.v_{i}\left(y_{j}, z_{j}\right)+\varepsilon_{i j}\right)=\beta \ln \left(y_{j}\right)+\alpha_{i} z_{j}+\varepsilon_{i j}$

Si se convierte en logaritmos naturales $y_{j}-t_{j}$ y $y_{j}$ de la ecuación 5 y se arreglan los términos, la función de probabilidad de respuesta del individuo $\mathrm{j}$ es:

$\operatorname{Pr} o b\left(\beta \ln \left(\frac{y_{j}-t_{j}}{y_{j}}\right)+\alpha z_{j}>-\varepsilon_{j}\right)$

$\mathrm{Al}$ igual que la función lineal, los componentes estocásticos y el parámetro $\alpha$ están agrupados. Si el término estocástico sigue una distribución normal tipificada, se mantienen los supuestos relacionados a este tipo de especificación y la probabilidad del encuestado de responder positivamente se expresa como:

$\operatorname{Pr} o b\left(S i_{j}\right)=\varphi\left(\frac{\beta \ln \left(\frac{y_{j}-t_{j}}{y_{j}}\right)+\alpha z_{i}}{\sigma}\right)=\varphi\left(X_{j} \beta^{*}\right)$ 
Donde $X_{j}^{\prime}$ es un vector compuesto por variables como el ingreso, el precio y de tipo socioeconómicas; $\beta^{*}$ es un vector de parámetros.

La especificación de utilidad con transformación Box-Cox en el ingreso se expresa por $y^{\lambda}=\frac{y^{\lambda}-1}{\lambda}$, tipo de especificación que supone que $y \sim N(X \beta$, $\sigma^{2} I_{n}$ ), el cual va de acuerdo con el modelo lineal. La función de utilidad se define sustituyendo $y^{\lambda}$ dentro de ella por $y$. La transformación resulta en $v_{i}\left(y_{j}, z_{i}\right)+\varepsilon_{i j}=\beta y_{i j}^{\lambda}+\alpha_{i} z_{j}+\varepsilon_{i j}$. Sin embargo, la forma que asume la función estará condicionada por el valor de $\lambda$. Cuando éste sea 0 la función se convertirá en la log-lineal; cuando sea 1 será lineal en el ingreso. La transformación Box-Cox supone que su parámetro es constante a través de las utilidades. Así, se define la probabilidad de respuesta afirmativa como:

$\operatorname{Pr} o b\left(S i_{j}\right)=\operatorname{Pr} o b\left[\beta \frac{\left(y_{j}-t_{j}\right)^{\lambda}-y_{j}^{\lambda}}{\lambda}+\alpha z_{j}>-\varepsilon_{j}\right]$

Donde $\alpha$ y $\varepsilon_{j}$ están agrupados. Pero si $\varepsilon_{j}$ sigue una distribución normal tipificada, la probabilidad de que el encuestado $\mathrm{j}$ responda de manera positiva se expresa:

$\operatorname{Pr} o b\left(S i_{j}\right)=\varphi\left(\frac{\beta\left[\frac{\left(y_{j}-t_{j}\right)^{\lambda}-y_{j}^{\lambda}}{\lambda}+\alpha z_{j}\right]}{\sigma}\right)$

Y tiene lugar un modelo probit.

La decisión de incorporar las variables descritas en el modelo probabilístico probit para las tres especificaciones de utilidad se basó en que fueran estadísticamente significativas para la prueba de razón de verosimilitud. Ésta se obtiene al restar el logaritmo de verosimilitud restringido y el logaritmo de verosimilitud sin restricción multiplicando el resultado por -2. También se utilizó el Criterio de Información Bayesiano (BIC, por sus siglas en inglés). De igual modo, la decisión de incluir las variables se basó en el que tuviera el menor valor del BIC.

\section{Resultados y discusión}

Los estadísticos descriptivos de cada una de las variables se muestran en el cuadro 4. 
Cuadro 4. Estadística descriptiva de las variables seleccionadas

\begin{tabular}{llcccc}
\hline Variable & Unidades & Media & S.D. & Mínimo & Máximo \\
\hline Ingreso & Pesos/mes & $\$ 10,143.99$ & $\$ 9,231.20$ & $\$ 500$ & $\$ 70,000$ \\
pers_gas & Personas & 1.8127 & 0.9572 & 1 & 6 \\
encuen_r & Binario & 0.7023 & 0.4576 & 0 & 1 \\
sabe_que & Binario & 0.7959 & 0.4033 & 0 & 1 \\
ser_am_c & Categórica & 3.0016 & 1.4487 & 0 & 6 \\
School & Categórica & 2.7378 & 1.178 & 0 & 5 \\
imp_gen & Categórica & 3.8781 & 0.3613 & 1 & 4 \\
\hline
\end{tabular}

Fuente: Elaboración propia con base en encuesta.

En el modelo probit estimado en sus tres versiones de función de utilidad para una prueba $X^{2}$ con siete grados de libertad, se rechaza la hipótesis nula de que los coeficientes estimados sean simultáneamente iguales a cero con un nivel de confianza del 99\%. Como medida de bondad de ajuste, y por ser el más utilizado en la literatura, se calculó el McFadden $\mathrm{R}^{2}$. En los tres modelos cayó dentro del intervalo de los resultados empíricos de los estudios de Mvc, aunque la bondad de ajuste resultó más baja para la especificación "función de utilidad logarítmica" y "la transformación Box-Cox". Los modelos tienen una capacidad de predicción de 72 a 80\%; para la especificación con función de utilidad lineal resulta más alta (cuadro 5).

Todos los coeficientes estimados del modelo probit en sus tres versiones de utilidad son estadísticamente significativos a los niveles usuales de confianza, a excepción de la escolaridad en la función log-lineal y la transformación Box-Cox. La variable principal, que es el precio, resultó con signo negativo para la especificación de la función de utilidad lineal. Así fue debido a que es una función de demanda; la probabilidad de aceptar pagar disminuye conforme se incrementa la tarifa propuesta. Lo anterior indica que conforme se incrementa el precio ofertado al entrevistado, menor es la probabilidad de que responda positivamente a la pregunta dicotómica. Las otras dos versiones, en su respectiva trasformación entre el ingreso y el precio propuesto, resultaron con el signo esperado, de acuerdo con Habb \& McConell (2002). El coeficiente que relaciona el precio ofrecido al individuo con su respectivo ingreso aumenta también porque se trata de formas funcionales más complejas, y el precio ofertado no es el único presente en la función de utilidad.

El resto de las variables arrojaron el signo esperado, con excepción del número de personas que aportan al gasto familiar y las variables "encuen_r" y "sabe_que". El que un mayor número de individuos aporte económicamente al hogar, no significa que la familia tenga mayor poder adquisitivo. Al estimar una regresión con variables interactivas, se aprecia que el coeficiente del precio con ingresos bajos es menor que el de ingresos altos. El resultado que se arroja es una DAP más 
alta para este último grupo. Así pues, la variable "precio" que interactúa con la variable dicotómica que representa ingresos bajos debe ser más alta que aquéllas con ingresos altos (cuadro 5). En la variable "encuen_r", las personas que contestaron correctamente dónde se encuentra la RBSL, tienen menor probabilidad de estar dispuestos a pagar, lo mismo que quienes saben que la RBSL es un área natural protegida.

Cuadro 5. Modelo de Elección Discreta Binario (probit)

\begin{tabular}{|c|c|c|c|c|c|c|c|c|}
\hline \multirow[b]{2}{*}{ Variables } & \multicolumn{4}{|c|}{ Función de utilidad lineal } & \multirow{2}{*}{$\begin{array}{c}\begin{array}{c}\text { Función } \\
\text { de utilidad } \\
\text { log-lineal }\end{array} \\
\text { Coeficiente }\end{array}$} & \multirow[b]{2}{*}{$P>Z$} & \multicolumn{2}{|c|}{$\begin{array}{c}\text { Transformación } \\
\text { Box-Cox }\end{array}$} \\
\hline & Coeficiente & $P>Z$ & Coeficiente & $P>Z$ & & & Coeficiente & $P>Z$ \\
\hline Constante & -0.1137 & 0.8541 & -0.0425 & 0.9462 & -0.2198 & 0.7094 & 0.138 & 0.821 \\
\hline monto-dap & $-0.0266^{\star * *}$ & 0.0000 & & & & & & 0.0000 \\
\hline $\begin{array}{l}\text { Dingresos } \\
\text { bajos xmonto dap }\end{array}$ & & & $-0.0275^{\star \star \star}$ & 0.0000 & & & & \\
\hline \multicolumn{9}{|l|}{$\begin{array}{l}\text { Dingresos } \\
\text { altos } \times \text { monto dap }\end{array}$} \\
\hline $\ln [(y-t) / y] / \sigma$ & & & $-0.0256^{\star \star \star}$ & 0.0000 & $47.6348^{* * *}$ & 0.0000 & & \\
\hline$\left[\left(\left(y_{j}-t_{j}\right)^{\lambda}-y^{\lambda}\right) / \lambda\right] / \sigma$ & & & & & & & $1.4314^{\star \star \star}$ & 0.0000 \\
\hline pers_gas & $-0.0013^{\star \star}$ & 0.0124 & $-0.0013^{\star \star}$ & 0.0115 & $-0.0020^{* * *}$ & 0.0009 & $-0.0019^{\star \star \star}$ & 0.0097 \\
\hline encuen_r & $-0.0015^{\star \star \star}$ & 0.0013 & $-0.0015^{\star \star \star}$ & 0.0014 & $-0.0019^{* * *}$ & 0.0002 & $-0.0018^{\star \star \star}$ & 0.0017 \\
\hline sabe_que & $-0.0014^{\star \star \star}$ & 0.0038 & $-0.0014^{\star \star \star}$ & 0.0038 & $-0.0021^{* * *}$ & 0.0000 & $-0.0019^{\star \star *}$ & 0.0004 \\
\hline ser_am_c & $0.0019^{\star \star \star}$ & 0.0004 & $0.0019^{\star \star \star}$ & 0.0004 & $0.0023^{\star \star \star}$ & 0.0000 & $0.0022^{\star \star \star}$ & 0.0000 \\
\hline Escolaridad & $0.1764^{\star \star \star}$ & 0.0008 & $0.1608^{\star \star \star}$ & 0.0046 & 0.0014 & 0.9778 & 0.0138 & 0.7919 \\
\hline imp_gen & $0.3981^{* *}$ & 0.0113 & $0.3900^{\star *}$ & 0.0137 & $0.2988^{* *}$ & 0.0400 & $0.3239^{\star \star}$ & 0.0321 \\
\hline Log-likelihood & -275.1245 & & -274.6845 & & -329.4146 & & -329.4146 & \\
\hline $\begin{array}{l}\text { Restricted log } \\
\text { likelihood }\end{array}$ & -369.4779 & & -369.4779 & & -369.4779 & & -369.4779 & \\
\hline McFadden $\mathrm{R}^{2}$ & 0.2553 & & 0.2566 & & 0.1084 & & 0.1961 & \\
\hline
\end{tabular}

*Los coeficientes son significativos al 10\%; ${ }^{* *}$ Los coeficientes son significativos al $5 \%$; ${ }^{* *}$ Los coeficientes son significativos al $1 \%$

Fuente: Elaboración propia con base en encuesta.

Los coeficientes estimados para las tres especificaciones de utilidad no cambian ni en signo ni en magnitud de manera considerable, y están más cerca de las funciones logarítmicas y de la transformación Box-Cox. Sin embargo, el nivel de significancia cambia en algunas variables. Tal es el caso de la función de utilidad lineal, resultando la variable "pers_gas" significativa al 5\%. En las otras dos funciones es del $1 \%$. Otra variable como la escolaridad fue significativa solamente para la primera versión de utilidad.

Para el modelo con la transformación Box-Cox en la función de utilidad, es importante mencionar que cuando $\lambda=1$, la función de utilidad es lineal en el ingreso $y$, cuando $\lambda=0$, es log-lineal en el ingreso. Con base en este razonamiento, 
cuando los valores se acercan a 1, la función se aproximaría a la linealidad y a cero a la log-lineal. Habb $\&$ McConell (2002) apuntan que el valor de $\lambda$ puede ser desconocido o predeterminado, por ello utilizamos la raíz cuadrada de $y$ (valor de $\lambda=0.5$ ), pues la función de utilidad no adoptaría la forma lineal ni la $\log$-lineal. De acuerdo con Buthmann (2011), si $\lambda=0.5$, entonces $\mathrm{y}^{\lambda} \cong \sqrt{\mathrm{y}}$.

Con respecto a la disposición a pagar (DAP), en el cuadro 6 se muestran las fórmulas para su cálculo con base en la mediana. Se recurre a las tres especificaciones mencionadas de la función de utilidad.

$\begin{gathered}\text { Cuadro 6. Fórmulas para calcular la DAP usando la mediana como medida } \\
\text { de tendencia central }\end{gathered}$
\begin{tabular}{lc}
\hline Función de utilidad & Modelos probit \\
\hline Lineal & $\alpha z_{j} / \beta$ \\
Log-lineal & $y-y \cdot \exp \left[-\left(\alpha z_{j} / \beta\right)\right]$ \\
Transformación Box-Cox & $y-\left[y^{\lambda}-\lambda \alpha z_{j} / \beta\right]^{1 / \lambda}$ \\
\hline Fuente: Elaboración propia con base en Habb \& McConell (2002).
\end{tabular}

Como se aprecia en el cuadro 7, la mediana de la DAP derivada de las funciones de utilidad del modelo probit se encuentra entre 69 y 108 pesos. Para fines comparativos, es importante señalar que los resultados probit y logit de la DAP son muy parecidos entre las mismas especificaciones de función de utilidad. Ahora bien, resultan más similares para la lineal, puesto que las funciones de densidad acumuladas logística y normal son muy similares (cuadros 7 y 9 del anexo). Las diferencias entre la log-lineal y la Box-Cox son ligeramente mayores debido a que, en la primera versión, el error ingresa exponencialmente en la función y en la Box-Cox y la expresión de la DAP es una transformación no lineal del error no observable.

Econometristas como Maddala (1983) y Greene (2003) señalan que los dos modelos son muy similares y que discrepan únicamente en la rapidez con la que las curvas se aproximan a los valores extremos. Así, la función logística es más "achatada" que la normal, porque ésta alcanza más rápido los valores extremos ( 0 y 1$)$.

Los resultados del presente trabajo se encuentran dentro de los límites de los estudios de valoración de preferencias expresadas sobre establecer el cobro de una cuota por entrar a los parques terrestres y bosques nacionales que muestra el cuadro 1. En este sentido, la probabilidad de cobrar una cuota adicional a la que pagan los individuos por entrar a la RBSLA y que se acceda a pagarla es consistente con la literatura de MvC sobre la preferencia de los individuos por la elección de pagar una tarifa por entrar a parques marinos y bosques. 
Por su parte, el valor económico adicional derivado de entrar a la RBSLA asciende a más de 26 millones de pesos (cuadro 7). Los resultados muestran que existen diferencias importantes cuando se consideran distintas especificaciones de utilidad y segmentación en el nivel de ingresos. Éstas han de considerarse al momento de establecer y tomar decisiones en materia de política ambiental.

Cuadro 7. Valor económico por acceder a la RBSLA con estimaciones del modelo probit

\begin{tabular}{lcccc}
\hline & \multicolumn{2}{c}{ Beneficio económico por persona } & \multicolumn{2}{c}{ Beneficios económicos totales } \\
Modelo & Pesos & Dólares & Pesos & Dólares \\
\hline $1^{*}$ & 71.86 & 5.69 & 27578441.23 & 2183566.21 \\
$2^{\star \star}$ & 69.27 & 5.48 & 26583452.57 & 2104786.43 \\
$2^{\star \star *}$ & 74.53 & 5.90 & 28603041.73 & 2264690.56 \\
$3^{\star \star \star *}$ & 107.62 & 8.52 & 41299558.74 & 3269957.15 \\
$4^{\star \star \star * *}$ & 100.56 & 7.96 & 38589911.84 & 3055416.61 \\
\hline
\end{tabular}

Notas: *DAP obtenida con función de utilidad lineal; **DAP con función lineal obtenida con ingresos bajos. ***DAP con función lineal obtenida con ingresos altos; ${ }^{* * \star *}$ DAP obtenida con función logarítmica; ${ }^{* \star * *}$ DAP obtenida con transformación Box-Cox. Tipo de cambio a 12.63 de 2010.

Fuente: Elaboración propia con base en la encuesta.

\section{Conclusiones}

El objetivo principal de la presente investigación fue estimar la mediana de la disposición a pagar (DAP) para así calcular una cuota adicional a la que CONANP fijó por acceder a la Reserva de la Biosfera Sierra de La Laguna. La metodología utilizada fue el Método de Valoración Contingente (MVC). Para ello, se estimaron tres versiones de función de utilidad con modelos de elección binarios como el probit. Las tres especificaciones de utilidad con base en las estimaciones de los modelos de oDC en su conjunto resultaron significativas al 99\% de confianza, de acuerdo con la prueba razón de verosimilitud.

Los resultados muestran que se pueden obtener beneficios económicos adicionales vía cobro de una mayor cuota que la establecida por CONANP por acceder a la RBSLA. De esta forma, los fondos recaudados se canalizarían a la mejora de los programas de manejo de la Reserva y, por ende, habría un sensible incremento en la conservación de la zona. Se trata de un patrimonio natural muy rico en biodiversidad; su preservación es materia relevante no sólo para los habitantes de Baja California Sur, sino para toda persona del exterior que desee visitar la Reserva.

En este sentido, el presente trabajo proporciona evidencia empírica pertinente que puede ser de utilidad a los tomadores de decisión encargados del uso y manejo de la RBSLA. El aumento de fondos económicos vía el cobro para ingresar a la Reserva, podría incentivar la ejecución de programas específicos de conservación 
dentro de la misma. No obstante, es preciso reconocer la presencia de factores que pueden inhibir este proceso, a saber: programaciones fallidas de visitas, falta de educación ambiental y políticas de desarrollo no sustentable, entre otros.

La DAP obtenida por el cobro adicional por entrar a la RBSLA representa, desde el punto de vista microeconómico, el excedente del consumidor, mismo que debe compararse con los costos de manejo de la Reserva para realizar el análisis costo-beneficio. La finalidad es que éste sirva de guía para tomar la decisión más viable. Actualmente el costo por acceder a la RBSLA es de 26.99 pesos. El estudio de Valoración Contingente muestra evidencia empírica de que los elaboradores de política pueden usarlo como punto de referencia para variar la cuota entre 155 y $296 \%$ a lo que adicionalmente pagan los usuarios.

\section{Referencias}

\section{Bibliohemerografía}

Adams, C., Seroa da Motta R., Arigoni Ortiz R., Reid, J., Ebersbach C. \& De Almeida Sinisgalli P. A. (2008). The Use of Contingent Valuation for Evaluating Protected Areas in the Developing World: Economic Valuation of Morro do Diablo State Park, Atlantic Rainforest, Sao Paulo State (Brazil). Ecological Economics, 66(2-3), 359-370.

Arin, T. \& Randall, A. K. (2002). Divers' Willingness to Pay to Visit Marine Sanctuaries: An Exploratory Study. Ocean and Coastal Management, 45(2), 171-183.

Arriaga, L. \& Ortega, A. (1988). La Sierra de La Laguna en Baja California Sur. México: Centro de Investigaciones Biológicas de Baja California A.C.

Arrow, K. \& Solow, R. (1993). Report of the NOAA Panel on Contingent Valuation: National Resource. Damage Assessments under the Oil Pollution Act of 1990, 58. Estados Unidos de América: Federal Register.

Bandara, R. \& Tisdell, C. (2004). The Net Benefit of Saving the Asian Elephant: A Policy and Contingent Valuation Study. Ecological Economics, 48(1), 93-107.

Baral, N., Stern, M. J. \& Bhattarai (2008). Contingent Valuation of Ecotourism in Appapurna Conservation Area, Nepal: Implications for Sustainable Park Finance and Local Development. Ecological Economics, 66(2-36), 218-227.

Bergstrom, J. Stoll, J. R. \& Randall, A. (1989). Information Effects in Contingent Markets. American Journal of Agricultural Economics, 71(3), 685-691. 
Boyle, K. \& Bishop, R. (1988). Welfare Measurements Using Contingent Valuation: A Comparison of Techniques. American Journal of Agricultural Economics. 70(1), 20-28.

Boyle, K., Bishop, R. \& Welsh, M. (1985). Starting Point Bias in Contingent Valuation Bidding Games. Land Economics, 61(21), 188-194.

Bishop, R. \& Heberlein, T. (1979). Measuring Values of Extra Market Goods: Are Indirect Measures Biased? American Journal of Agriculture Economics, 61(5), 926-930.

Breceda, A., Castellanos, A., \& Mercado, C. (1996). Programa de Manejo de la Reserva de la Biosfera Sierra La Laguna. México: Centro de Investigaciones Biológicas del Noroeste, S. C.

Brookshire, D. (1976). The Valuation of Aesthetic Preferences. Journal of Environmental Economics and Management, 3(4), 325-346.

Carson, R. \& Haneman, M. W. (2005). Contingent Valuation. Handbook of Environmental Economics, 2, 821-396.

Carson, R. (2000). Contingent Valuation: A User's Guide. Environmental Science and Technology, 34(8), 1413-1418.

Casey, J. F. (2006). Are Tourists Willing to Pay Additional Fees to Protect Corals in Mexico. Estados Unidos de América.

Chase, L., Lee, R. D., Schulze, W. \& Anderson, D. J. (1998). Ecotourism Demand and differential Pricing of National Park Access in Costa Rica. Land Economics, 74(4), 466-482.

Conanp. (2010). Oficio solicitado de los registros de acceso de turistas a la Reserva de la Biosfera Sierra La Laguna. México: CONANP.

conanp. (2007). Programa de Turismo en Áreas Protegidas 2007-2012. México: conanp.

Conanp. (2003). Programa de Manejo Reserva de la Biosfera Sierra La Laguna, México: SEMARnat.

Cummings, R. Ganderton, P. T., \& MacGuckin, T. (1994). Substitution Effects in cvm Values. American Journal of Agriculture Economics, 76(2), 205-214.

cvgm. (1997). Valuing Ground Water: Economic Concepts and Approaches. Washington: The National Academies Press.

Dudley, N. (2008). Directrices para la aplicación de las categorias de gestión de áreas protegidas. Suiza: UICN. 
Ghanbarpour, M., Sajjadi, Sh., Hajiseyedjavadi, S., \& Xiangming, Ch. (2011). Investigation of Visitors' Participation and Willingness to Pay for the Baba Aman Recreational Park, Iran. Research Journal of Environmental and Earth Sciences, 3(6), 722-728.

Greene, W. (2003). Econometric Analysis. Nueva Jersey: Prentice-Hall.

Habb, T. \& McConnell, K. (2002). Valuing Environmental and Natural Resources: The Econometrics of Non-Market Valuation. Reino Unido: Edward Elgar Publishing.

Hanemann, W. M. (1994). Valuing the Environment through Contingent Valuation. Journal of Economic Perspectives, 8(4), 19-43.

Hanley, N. (1988). Using Contingent Valuation to Value Environmental Improvements. Applied Economics, 20(4), 541-549.

Hernández, V. (2009). Valoración económica del Parque Nacional Bahía de Loreto a través de los servicios de recreación de pesca deportiva. Región y Sociedad, 44(21), 195-223.

Hoehn, J. \& Randall, A. (1987). A Satisfactory Benefit Cost Indicator from Contingent Valuation. Journal of Environmental Economics and Management, 14(3), 226-247.

INEgI. (2005). Conteo de Población y Vivienda 2005. México: INEGI.

Inegr. (2014). Sistema de Cuentas Nacionales de México: Cuenta satélite del turismo de México 2012. México: INEGI.

Israngkura, A., (1998). Environmental Valuation: An Entrance Fee System for National Parks in Thailand. Singapur: Economy and Environment Program for Southeast Asia.

Kontoleon, A. \& Swanson, T. (2003). The Willingness to Pay for Property Rights for the Giant Panda: Can a Charismatic Species Be an Instrument for Nature Conservation? Land Economics, 79(4), 483-499.

Krug, W., Suich, H., \& Haimbodi, N. (2002). Park Pricing and Economic Efficiency in Namibia. Discussion Paper núm. 45.

Krutilla, J. V. (1967). Conservation Reconsidered. The American Economic Review, 57, 777-786.

Lindsey, G. Holmes, A. (2002). Tourist Support for Marine Protection in Nha Trang, Vietnam. Journal of Environmental Planning and Management, 45(4), 461-480.

Maddala, G. S. (1983). Limited-Dependent and Qualitative Variables in Econometrics. Reino Unido: Cambridge University Press. 
Milon, J. W. (1989). Contingent Valuation Experiments for Strategic Behavior. Journal of Environmental Economics and Management, 17(3), 293-308.

Mitchell, R. C. \& Carson, R. T. (1989). Using Surveys to Value Public Goods: The Contingent Valuation Method. Washington: Resources for the Future.

Mohd Rusli, Y. (2009). Contingent Valuation of Ecotourism in Marine Parks, Malaysia: Implication for Sustainable Marine Park Revenue and Ecotourism Management. World Applied Sciences Journal, 7(12), 1474-1481.

Mohd Rusli, Y., Seddigheh Arab, A., Alias, R., Zaiton, S. \& Ahmad, S. (2009). Recreational Demand in Bird Sanctuary: The Case of Kapar Bird Sanctuary, Kelang, Malaysia. International Journal of Business and Management, 4(12), 99-111.

Muñoz-Piña, C. (2003). Demanda turistica del Parque Marino Cabo Pulmo. México: Ine.

oECD. (1995). The Economic Appraisal of Environmental Projects and Policy: A Practical Guide. París.

oмT. (2012). Panorama OMT del turismo internacional, Edición 2012. Madrid: UNwTO.

Ortega, A. (1992). Uso y manejo de los recursos naturales en la Sierra de La Laguna, Baja California Sur, México: WwF.

Osorio, J. \& Correa, F. J. (2009). Un análisis de la aplicación empírica del Método de Valoración Contingente. Semestre Económico, 12(25), 11-30.

Padilla, A. G. (1988). Historia geológica y paleoecología. En L. Arriaga y A. Ortega (Eds.). La Sierra La Laguna de Baja California Sur. México: сів.

Pérez, G. (1998). Turismo en las Áreas Naturales Protegidas: Valuación económica de los beneficios recreativos del Santuario de El Campanario. En Benítez, H., Vega, E., Peña, A. \& Ávila S. (Eds.). Aspectos económicos sobre la biodiversidad de México. México: Conabio/semarnap/Ine.

Peters, H. \& Hawkins, J. (2009). Access to Marine Parks: A Comparative Study in Willingness to Pay. Ocean and Costal Management, 52, 219-228.

Ransom, K. \& Mangi, S. (2010). Valuing Recreational Benefits of Coral Reefs: The Case of Mombasa Marine National Park and Reserve, Kenya. Environmental Management, 45(1), 145-154.

Riera, P. (1994). Manual de Valoración Contingente. Madrid: Instituto de Estudios Fiscales.

Riley, E. (2006). A Willingness to Pay Study for Park Fees: Quill/ Boven National Park St Eustatius Marine Park St Eustatius. Antilles: St Eustatius National Parks Foundation. 
Rivera, M. \& Muñoz, C. (2005). Tarifas y arrecifes: instrumentos económicos para áreas naturales protegidas marinas en México. Gaceta Ecológica, 75, 19-34.

Romo, J. L. (1998). Valoración económica de la migración de las Mariposas Monarcas. En H. Benítez, E. Vega, A Peña, S. Ávila (Eds.). Aspectos económicos sobre la biodiversidad conaBıo. México: SEMARNAP/INE.

Rowe, R., D’Arge, R. \& Brookshire, D. (1980). An Experiment on the Economic Value of Visibility. Journal of Environmental Economics and Management, 7(1), 1-19.

Samdin, Z. (2008). Willingness to Pay in Taman Negara: A Contingent Valuation Method. Journal of Economics and Management, 2(1), 81-94.

Sectur. (2013). Plan Nacional de Desarrollo 2013-2018, Gobierno de la República, Programa Sectorial de Turismo. México: 88.

Thayer, M. (1981). Contingent Valuation Techniques for Assessing Environmental Impacts: Further Evidence. Journal of Environmental Economics and Management, 8(1), 27-44.

Train, K. (2009). Discrete Choice Methods with Simulation. Cambridge: Cambridge University Press.

Tsi, E. A., Nji, A. \& Muhlenberg, M. (2008). The Willingness to Pay (wTP) for the Conservation of Wild Animals: Case of the Derby Eland (Taurotragus derbianus gigas) and the African Wild Dog (Lycaon pictus) in North Cameroon African. Journal of Environmental Science and Technology, 2(3), 51-58.

UNEP-wCMC. (2012) Protected Planet Report 2012. United Nations.

Wang, Peng-Wei \& Jing-Bo J. (2012). Tourists' Willingness to Pay for Biodiversity Conservation and Environment Protection, Dalai Lake Protected Area: Implications for Entrance Fee and Sustainable Management. Ocean and Coastal Management, 62(4), 24-33.

Whitehead, J. \& Blomquist, G. (1991). Measuring Contingent Values for Wetlands: Effects of Information about Related Environmental Goods. Water Resources Research, 27(10), 2523-2531.

\section{Recursos electrónicos}

Buthmann, A. (2012). Making Data Normal Using Box-Cox Power Transformation. Recuperado de http://www.isixsigma.com/tools-templates/normality/making-data-normal-using-box-coxpower-transformation/

CONANP. (2011). http://www.conanp.gob.mx/ 
CONAFOr. (2013). Áreas Protegidas Decretadas. SEMarnat. Recuperado de http://www.conanp .gob.mx/que_hacemos/

DoF. (2012). Ley Federal de Derechos. México. Recuperado de http://www.diputados.gob.mx/ LeyesBiblio/pdf/107.pdf

Dof. (1988). Ley General de Equilibrio Ecológico y Protección al Ambiente. México. Recuperado de http://www.diputados.gob.mx/LeyesBiblio/index.htm

Ecotourism Society. (1998). Ecotourism Statistical Fact Sheet. Recuperado de http://www .ecotourism.org/textfiles/stats.text

SECTUR. (2014). Sistema Nacional de la Información Estadistica del Sector Turismo de México. Recuperado de http://www.datatur.sectur.gob.mx/

\section{Anexo}

Cuadro 8. Modelo de elección discreta binario (logit)

\begin{tabular}{|c|c|c|c|c|c|c|c|c|}
\hline \multirow[b]{2}{*}{ Variables } & \multicolumn{4}{|c|}{ Función de utilidad lineal } & \multicolumn{2}{|l|}{$\begin{array}{l}\text { Función } \\
\text { de utilidad } \\
\text { log-lineal }\end{array}$} & \multicolumn{2}{|c|}{$\begin{array}{c}\text { Transformación } \\
\text { Box-Cox }\end{array}$} \\
\hline & Coeficiente & $P>Z$ & Coeficiente & $P>Z$ & Coeficiente & $P>z$ & Coeficiente & $P>Z$ \\
\hline Constante & -0.1289 & 0.9027 & -0.007 & 0.9948 & -0.1916 & 0.8443 & 0.3958 & 0.702 \\
\hline monto-dap & $-0.0457^{\star \star \star}$ & 0.0000 & & & & & & \\
\hline $\begin{array}{l}\text { Dingresos } \\
\text { bajos×monto dap }\end{array}$ & & & $-0.0472^{* \star *}$ & 0.0000 & & & & \\
\hline $\begin{array}{l}\text { Dingresos } \\
\text { altos } \times \text { monto dap }\end{array}$ & & & $-0.0441^{* \star *}$ & 0.0000 & & & & \\
\hline $\ln [(y-t) / y] / \sigma$ & & & & & $87.2092^{* * *}$ & 0.0000 & & \\
\hline$\left[\left(\left(y_{j}-t_{j}\right)^{\lambda}-y^{\lambda}\right) / \lambda\right] / \sigma$ & & & & & & & $2.5161^{\star * \star}$ & 0.0000 \\
\hline pers_gas & $-0.0023^{*}$ & 0.0518 & $-0.0024^{* *}$ & 0.0500 & $-0.0039^{* *}$ & 0.0159 & $-0.0037^{*}$ & 0.0545 \\
\hline encuen_r & $-0.0030^{\star \star}$ & 0.0144 & $-0.0030^{* *}$ & 0.0147 & $-0.0040^{\star \star *}$ & 0.0077 & $-0.0038^{\star *}$ & 0.0189 \\
\hline sabe_que & $-0.0029^{\star *}$ & 0.023 & $-0.0030^{* *}$ & 0.0227 & $-0.0041^{* * *}$ & 0.0013 & $-0.0038^{\star * \star}$ & 0.0062 \\
\hline ser_am_c & $0.0033^{\star \star \star}$ & 0.0044 & $0.0032^{\star \star *}$ & 0.0047 & $0.0039^{* * *}$ & 0.0006 & $0.0037^{\star \star \star}$ & 0.0011 \\
\hline Escolaridad & $0.3063^{\star \star \star}$ & 0.0010 & $0.2792^{* \star *}$ & 0.0057 & -0.007 & 0.9373 & 0.0179 & 0.8437 \\
\hline imp_gen & $0.6611^{\star *}$ & 0.0135 & $0.6471^{* *}$ & 0.0166 & $0.4676^{* *}$ & 0.0491 & $0.5149^{\star *}$ & 0.0430 \\
\hline Log-likelihood & -274.9442 & & -274.5624 & & -328.3441 & & -328.3441 & \\
\hline $\begin{array}{l}\text { Restricted log } \\
\text { likelihood }\end{array}$ & -369.4779 & & -369.4779 & & -369.4779 & & -369.4779 & \\
\hline McFadden $\mathrm{R}^{2}$ & 0.2558 & & 0.2569 & & 0.1113 & & 0.1979 & \\
\hline
\end{tabular}

$\overline{ }$ *Los coeficientes son significativos al 10\%; ${ }^{\star *}$ Los coeficientes son significativos al $5 \%$; ${ }^{\star \star *}$ Los coeficientes son significativos al $1 \%$.

Fuente: Elaboración propia con base en encuesta.

Cuadro 9. Valor económico por acceder a la RBSLA con estimaciones del modelo logit

M. A. Almendarez-Hernández, I. Sánchez-Brito, M. V. Morales Zárate, C. A. Salinas-Zavala | Propuesta de cuotas para conservación de un área natural protegida de México | Perfiles Latinoamericanos, 24(47) | FLACso México | pp. 95-120 


\begin{tabular}{|c|c|c|c|c|}
\hline \multirow{2}{*}{ Modelo } & \multicolumn{2}{|c|}{ Beneficio económico por persona } & \multicolumn{2}{|c|}{ Beneficios económicos totales } \\
\hline & Pesos & Dólares & Pesos & Dólares \\
\hline $1^{*}$ & 71.63 & 5.67 & 27488806.33 & 2176469.23 \\
\hline $2^{\star \star}$ & 69.22 & 5.48 & 26562414.74 & 2103120.72 \\
\hline $2^{\star \star \star}$ & 74.10 & 5.87 & 28435856.68 & 2251453.42 \\
\hline $3^{\star \star \star \star}$ & 103.41 & 8.19 & 39685101.79 & 3142129.99 \\
\hline $4^{\star \star \star \star \star}$ & 97.47 & 7.72 & 37406350.18 & 2961706.27 \\
\hline
\end{tabular}

Notas: *DAP obtenida con función de utilidad lineal; ${ }^{* *}$ DAP con función lineal obtenida con ingresos bajos. ${ }^{* * *}$ DAP con función lineal obtenida con ingresos altos; ${ }^{* \star \star \star D A P}$ obtenida con función logarítmica; ${ }^{\star \star \star \star \star}$ DAP obtenida con transformación Box-Cox. Tipo de cambio a 12.63 de 2010.

Fuente: Elaboración propia con base en encuesta.

Recibido el 17 de enero de 2014. Aceptado el 16 de junio de 2015. 